\title{
A TEST OF THE EIKONAL APPROXIMATION IN HIGH-ENERGY (e,e'p) SCATTERING
}

\author{
A. Bianconi and M. Radici \\ Dipartimento di Fisica Nucleare e Teorica, Università di Pavia, and \\ Istituto Nazionale di Fisica Nucleare, Sezione di Pavia, \\ v. Bassi 6, 27100 Pavia, Italy
}

\begin{abstract}
The Glauber method is extensively used to describe the motion of a hadronic projectile in interaction with the surrounding nuclear medium. One of the main approximations consists in the linearization of the wave equation for the interacting particle. We have studied the consequences of such an assumption in the case of the ${ }^{12} \mathrm{C}\left(\mathrm{e}, \mathrm{e}^{\prime} \mathrm{p}\right){ }^{11} \mathrm{~B}^{*}$ reaction at high proton momenta by comparing the results with the predictions obtained when all the ingredients of the calculation are unchanged but the second-order differential equation for the scattered wave, which is solved exactly for each partial wave up to a maximum of 120 spherical harmonics. We find that the Glauber cross section is always larger by a factor $10 \div 20 \%$, even at vanishing missing momenta. We give a quantum-mechanical explanation of this discrepancy. Nevertheless, a good correlation is found between the two predictions as functions of the missing momentum, especially in parallel kinematics.
\end{abstract}

The problem of the validity of the Glauber approximation [1] in (e,e'p) scattering from finite nuclei is receiving more and more interest in relation with the experiments planned at CEBAF, where the proton momentum can be larger than $1 \div 2 \mathrm{GeV} / \mathrm{c}$. At large missing momenta of the recoil the details of the short-range nucleon-nucleon interaction are expected to show up [2], but the few experimental data available [3] still prevent from putting stringent constraints on the theoretical models. On the other side, at moderate missing momenta an accuracy within $10 \%$ is required to unambigously identify exotic effects like Colour Transparency, if any [4].

The recent NE18 experiment [5] has shown that calculations based on the standard Glauber approximation overestimate the cross section even at vanishing missing momenta, unless some phenomenological corrections are introduced. Various suggestions have been made both on the way of analyzing the NE18 data [6, 7] and on how to improve the Glauber method [8]. Starting from a different point of view, we show that the latter is affected by an intrinsic systematical error, which can be relevant at the kinematics 
explored at CEBAF but seems anyway to be predictable and perhaps can be corrected for.

Traditionally, the Glauber method has been extensively used in the analysis of the proton-nucleus scattering data [9, 10], where it gives good, or at least some of the best available, fits to the scattering distributions. However, the generalization to the (e,e $\mathrm{p}$ ) reaction is not straightforward. In fact, even if the hadronic content of the final state is similar, the inelastic nature of the electromagnetic proton emission makes the kinematical situation rather different.

The basic ingredient to describe the knockout process for an exclusive scattering in the framework of DWIA, is the scattering amplitude [11, 12

$$
J_{\alpha}^{\mu}(\boldsymbol{q})=\int \mathrm{d} \boldsymbol{r} \mathrm{d} \sigma \mathrm{e}^{\mathrm{i} \boldsymbol{q} \cdot \boldsymbol{r}} \chi^{(-) *}(\boldsymbol{r}, \sigma) \hat{J}^{\mu}(\boldsymbol{q}, \boldsymbol{r}, \sigma) \Psi_{\alpha}(\boldsymbol{r}, \sigma)
$$

where $\boldsymbol{q}$ is the momentum transfer, $\chi^{(-)}$is the distorted wave function of the knockout nucleon and $\Psi_{\alpha}$ is the single-particle bound state wave function depending on the quantum numbers $\alpha$ of the hole. For sake of simplicity, in this work we have focussed on the longitudinal component of the current operator, retaining just the leading order $O(1)$ in the nonrelativistic expansion and neglecting the nucleon form factor, i.e. substituting $\hat{J}^{\mu}$ with the identity operator. The cross section becomes, therefore, proportional to

$$
\left|\int \mathrm{d} \boldsymbol{r} \mathrm{d} \sigma \mathrm{e}^{\mathrm{i} \boldsymbol{q} \cdot \boldsymbol{r}} \chi^{(-) *}(\boldsymbol{r}, \sigma) \Psi_{\alpha}(\boldsymbol{r}, \sigma)\right|^{2} \equiv S_{\alpha}^{\mathrm{D}}(\boldsymbol{q})
$$

which can be identified as the "distorted" spectral density $S_{\alpha}^{\mathrm{D}}$ [13] at the missing energy corresponding to the knockout hole $\alpha$.

The distorted wave function $\chi^{(-)}$is solution of the Schrödinger equation

$$
\left(-\frac{\hbar^{2}}{2 m} \nabla^{2}+V\right) \chi=E_{\mathrm{cm}} \chi,
$$

where $m$ is the reduced mass of the nucleon in interaction with the residual nucleus, $E_{\mathrm{cm}}$ is its kinetic energy in the $\mathrm{cm}$ system and $V$ in principle includes a nonlocal energydependent optical potential effectively describing the residual interaction. The incomingwave boundary conditions to be imposed on the solution of eq. (3) correspond to the well known feature of the quantum-mechanical problem for a wave crossing a potential barrier, where the asymptotic stationary conditions require the incoming unitary flux to be splitted in a reflected one and in an outgoing one.

The proper way of solving eq. (3) is to expand $\chi^{(-)}$in partial waves, solve the secondorder differential equation wave by wave, sum the solutions up to a certain maximum angular momentum $L_{\max }$ which satisfies an ad hoc convergency criterion for eq. (1). From now on, this procedure will be referred to as method I. So far, it has been applied to proton momenta below $0.3 \mathrm{GeV} / \mathrm{c}$ [12], where convergency is reached for $L_{\max }<50$. In this work we have extended it to larger proton momenta, i.e. $p \sim 1 \div 2 \mathrm{GeV} / \mathrm{c}$. A maximum $L_{\max }=120$ has been used and we have checked that the results become already stable with $L_{\max }=100$. 
At high energies the Glauber method [1] offers an alternative way (from now on method II) of solving eq. (3) by linearizing it along the propagation axis $\hat{z}$ :

$$
\begin{aligned}
\boldsymbol{r} & \equiv z \frac{\boldsymbol{p}}{p}+\boldsymbol{b} \\
\nabla^{2} & \simeq \frac{\partial^{2}}{\partial z^{2}} \\
\left(\frac{\partial^{2}}{\partial z^{2}}+p^{2}\right) & =\left(\frac{\partial}{\partial z}+\mathrm{i} p\right) \cdot\left(\frac{\partial}{\partial z}-\mathrm{i} p\right) \\
& \simeq 2 \mathrm{i} p \cdot\left(\frac{\partial}{\partial z}-\mathrm{i} p\right)
\end{aligned}
$$

where $\boldsymbol{b}$ is the impact parameter describing the degrees of freedom transverse to the motion of the struck particle with momentum $\boldsymbol{p}$. With this approximation eq. (3) becomes

$$
\left(\frac{\partial}{\partial z}-\mathrm{i} p\right) \chi=\frac{1}{2 \mathrm{ip}} V \chi
$$

The standard boundary condition applied requires that asymptotically $\chi \rightarrow 1$, which corresponds to an incoming unitary flux of plane waves. In contrast to method I no reflected flux is taken into account that is created by the potential of the nucleon-nucleon interaction. Because of the different phases, there is a destructive interference between the reflected and the incoming waves, which eq. (7) neglects thus leading to an overestimation of eq. (2). In particular, the approximation of eq. (6), upon which the eq. (7) is based, can be expected to produce most of the discrepancy at very small angles $\gamma$ between $\boldsymbol{q}$ and $\boldsymbol{p}$, typically in parallel kinematics. On the other side, at large $\gamma$ and large transverse missing momenta the approximation of eq. (5) is expected to play the major role.

A direct comparison with the exact solutions of eq. (3), summed up to $L_{\max }$, is needed to get a quantitative answer out of these qualitative considerations. The need for considering corrections beyond the eikonal approximation of the Glauber method is a well known problem in high-energy elastic proton scattering on nuclei [10, 14, 15]. One basic outcome of these studies is that competing effects tend to cancel each other at small deflection angles [14, while can give important corrections at larger angles 15. This fact suggests that also in $\left(\mathrm{e}, \mathrm{e}^{\prime} \mathrm{p}\right)$ reactions it could be interesting to compare deviations from the eikonality with other kinds of corrections to the Glauber model. This is the aim of a more general work, whose preliminary results we present in this communication.

Here, we have considered the ${ }^{12} \mathrm{C}\left(\mathrm{e}, \mathrm{e}^{\prime} \mathrm{p}\right){ }^{11} \mathrm{~B}^{*}$ reaction both in parallel and perpendicular kinematics for the proton momentum $p$ up to $2 \mathrm{GeV} / \mathrm{c}$ and the momentum transfer $q=1.4 \mathrm{GeV} / \mathrm{c}$. When not explicitely mentioned, the bound state $\Psi_{\alpha}$ must be understood as the solution of the Woods-Saxon potential of Comfort and Karp 16 with the quantum numbers $\alpha$ of the $s$ wave. As an exploratory calculation, we have neglected the contribution to $V$ in eq. (3) coming from the Coulomb potential to avoid numerical problems related to the high angular momenta required. Therefore, in proper terms the results 
presented here refer to the $\left(e, e^{\prime} n\right)$ reaction. $V$ is an optical potential of the form

$$
\begin{aligned}
V(r) & =(U+\mathrm{i} W) \frac{1}{1+\mathrm{e}^{\frac{r-R}{a}}} \\
& \equiv(U+\mathrm{i} W) \rho(r),
\end{aligned}
$$

with $R=1.2 \times A^{1 / 3} \mathrm{fm}$ and $a=0.5 \mathrm{fm}$. The nuclear density $\rho(r)$ defined in eq. (8) is normalized such that $\rho(0)=1$.

No phenomenological optical potential is available at the energies here considered. Therefore, the parameters $U, W$ can only be guessed. Two guidelines can help in this case. On one side, according to the Glauber model the imaginary part should scale as $W \sim p / 10 \mathrm{MeV}$, while $U / W$ should equal the ratio between the real and the imaginary parts of the average proton-nucleon forward-scattering amplitude, which is expected to be $\leq 0.5$ in the considered kinematics 17 . On the other side, assuming that the absorption due to the final state interactions, observed in the NE18 experiment in the context of a semi-inclusive $\left(\mathrm{e}, \mathrm{e}^{\prime} \mathrm{p}\right)$, is reasonable also for a completely exclusive knockout, then $U, W$ should be better taken about half of the previous values. We have considered several choices, including the unphysical but interesting cases of a completely real $(W=0)$ or imaginary $(U=0)$ potential.

By comparing the predictions of methods I and II for eq. (2) at $\boldsymbol{p}=\boldsymbol{q}$ we find that in the range $0.6 \leq p \leq 2 \mathrm{GeV} / \mathrm{c}$ the results are $p$-independent if $U, W$ are linear functions of $p$. This is a well known feature of the Glauber method at high energy proton-nucleus scattering. Hence, the previous findings suggest that, at least for small missing momenta, starting from $p \sim 0.6 \mathrm{GeV} / \mathrm{c}$ on we are already in a "Glauber regime". Therefore, in the following whenever the kinematics is parallel, we employ an optical potential of the form $(U+\mathrm{i} W) \rho(r) p / p_{\mathrm{o}}$, where $p_{\mathrm{o}}=1.4 \mathrm{GeV} / \mathrm{c}$.

Figs. 1 and 2 show the distorted spectral density of eq. (2) in parallel kinematics for missing momenta in the range $0 \leq p_{\mathrm{m}}=p-q \leq 600 \mathrm{MeV} / \mathrm{c}$. The dashed line represents the result with no final state interaction (PWIA), which is of course identical in both methods. The solid and the dotted lines are the results of method I and II, respectively. The two curves are rather well correlated in all the range of $p_{\mathrm{m}}$ here explored. But a systematic discrepancy is evident, even at $p_{\mathrm{m}} \sim 0$, which is roughly proportional only to the imaginary part of the optical potential, as it can be realized by inspecting curves in fig. 1 labeled by (a) $(U=100 \mathrm{MeV}, W=0)$ and (b) $(U=0, W=100 \mathrm{MeV})$, or in fig. 2 by (a) $(U=20, W=50 \mathrm{MeV})$ and $(\mathrm{b})(U=50, W=150 \mathrm{MeV})$. This fact suggests that the origin of the discrepancy should be ascribed to the linearization of eq. (3) implied by the approximation of eq. (6).

It is interesting also to explore the role of the Final State Interactions (FSI), which seem to have a nontrivial structure especially at high missing momenta in perpendicular kinematics. To accomplish this, we rewrite eq. (2) as

$$
\begin{aligned}
S_{\alpha}^{\mathrm{D}}(\boldsymbol{q}) & \sim|\mathrm{PWIA}+\mathrm{FSI}|^{2} \\
& =|\mathrm{PWIA}|^{2}+|\mathrm{FSI}|^{2}+2 \mathrm{Re}\left(\mathrm{PWIA} \cdot \mathrm{FSI}^{*}\right)
\end{aligned}
$$


and we compute the single contributions separately in the framework of method I. In fig. 3 the $\mid$ PWIA $\left.\right|^{2}, \mid$ FSI $\left.\right|^{2}$ and the total contribution $S_{\alpha}^{\mathrm{D}}$ are shown as functions of the angle $\gamma$ between $\boldsymbol{q}$ and $\boldsymbol{p}$ using a strong unrealistic optical potential $(U=50, W=150 \mathrm{MeV})$ to emphasize the role of FSI. At small angles the PWIA is the dominant contribution. The interference is negative and is responsible for the familiar damping observed in all electron scattering data. Near $\gamma \sim 15^{\circ}$ the angular distribution has a dip (partially filled if the real part $U$ of the optical potential is non vanishing), because the interference is roughly equal and opposite to the sum of $|\mathrm{PWIA}|^{2}$ and $|\mathrm{FSI}|^{2}$. At large angles the distribution basically coincides with $|\mathrm{FSI}|^{2}$.

To better specify this last issue let's consider the fig. 4, where the angular distribution $S_{\alpha}^{\mathrm{D}}$ is plotted in the same conditions as in fig. 3 but substituting inside $\Psi_{\alpha}$ the shellmodel wave function of the Woods-Saxon type with a harmonic oscillator with the same quantum numbers. In this case the dashed line, i.e. the PWIA contribution, falls down monotonously and quickly just after the Fermi momentum $\left(221 \mathrm{MeV} / \mathrm{c}\right.$ at $\left.\gamma \sim 10^{\circ}\right)$. Any diffractive pattern must therefore be ascribed to the FSI term. This remark, less obvious in the case of fig. 3 because of the richer structure of PWIA, is a common feature of both approaches, the method I (solid line) and the method II (dotted line). They appear correlated similarly to the case of the parallel kinematics.

The oscillatory diffractive trend of $S_{\alpha}^{\mathrm{D}}$ at large angles is reminiscent of the angular distribution for proton-nucleus scattering [9]. Thus, the natural interpretation is that the ejected proton is testing coherently the residual nucleus. This is possible only in a completely exclusive reaction, i.e. where the residual nucleus doesn't fragment. Any measurement of an energy-integrated distribution, i.e. of a semi-inclusive (e,e'p) reaction [6, 17, 18, 19], looses this information on the structure of the $A-1$ system and tests just the average behaviour of the single ejected nucleon.

We have critically analyzed some aspects of the Glauber approach to the description of the propagation of a particle through the nuclear medium in the case of the ${ }^{12} \mathrm{C}\left(\mathrm{e}, \mathrm{e}^{\prime} \mathrm{p}\right){ }^{11} \mathrm{~B}^{*}$ reaction. The basic approximation of linearizing the wave equation for the projectile has been shown to be responsible for a systematic discrepancy with respect to the results obtained from its proper solution. This fact could be relevant at the CEBAF kinematics, particularly at small missing momenta. At very large values of the transverse missing momenta the FSI give the dominant contribution to the cross section and show a diffractive pattern, which can naturally be interpreted as a coherent diffractive scattering between the ejected proton and the residual nucleus. To verify this prediction a completely exclusive $\left(\mathrm{e}, \mathrm{e}^{\prime} \mathrm{p}\right)$ reaction is needed, where the residual nucleus is in a well specified state.

We would like to thank prof. S. Boffi for many discussions and for his continuous interest in this work, and dr. F. Cannata [20] for having originally suggested the idea upon which this work is based.

\section{References}


[1] R.J. Glauber in: Lectures in Theoretical Physics, vol. 1, eds. W. Brittain and L.G. Dunham. Interscience Publ., N.Y., 1959;

R.J. Glauber and G. Matthiae, Nucl. Phys. B21, 135 (1970).

[2] C. Mahaux and R. Sartor, Adv. Nucl. Phys. 20, 1 (1991);

S.C. Pieper, R.B. Wiringa and V.R. Pandharipande, Phys. Rev. C46, 1741 (1992);

H. Müther and W.D. Dickhoff, Phys. Rev. C49, R17 (1994).

[3] I. Bobeldijk et al., Phys. Rev. Lett., 73, 2684 (1994);

K.I. Blomqvist et al., Phys. Lett., B344, 85 (1995).

[4] L. Frankfurt, M. Strikman and G.A. Miller, Comm. Nucl. Part. Phys. 21, 1 (1992); N.N. Nikolaev, Int. J. Mod. Phys. E3, 1 (1994);

A.Bianconi, S.Boffi and D.E.Kharzeev, Yadernaya Fizika 57, 1732 (1994).

[5] N.C.R. Makins et al. (the NE18 Collaboration), Phys. Rev. Lett 72, 1986 (1994).

[6] N.N. Nikolaev, A. Szczurek, J. Speth, J. Wambach, B.G. Zakharov and V.R. Zoller, Phys.Rev. C50, R1296 (1994).

[7] N.N. Nikolaev, A. Szczurek, J. Speth, J. Wambach, B.G. Zakharov and V.R. Zoller, Nucl.Phys A582, 665 (1995).

[8] V.R.Pandharipande and S.C.Pieper, Phys. Rev. C45, 791 (1992).

[9] R.H. Bassel and C. Wilkin, Phys. Rev. 174, 1179 (1968).

[10] G.D. Alkhazov, S.I. Belostotsky and A.A. Vorobyev, Phys. Rev. C42, 89 (1978).

[11] S. Frullani and J. Mougey, Adv. Nucl. Phys., vol. 13, eds. J.W. Negele and E. Vogt (1984).

[12] S. Boffi, C. Giusti and F.D. Pacati, Phys.Rep. 226, 1 (1993).

[13] S. Boffi, C. Giusti and F.D. Pacati, Nucl. Phys. A386, 599 (1982).

[14] S. Wallace, Phys. Rev. C12, 179 (1975).

[15] S.A. Gurvitz, Y. Alexander and A.S. Rinat, Ann. Phys. 98, 346 (1976).

[16] J.R. Comfort and B.C. Karp, Phys. Rev. C21, 2162 (1980).

[17] C. Lechanoine-LeLuc and F. Lehar, Rev. Mod. Phys. 65, 47 (1993).

[18] A. Bianconi, S. Jeschonnek, N.N. Nikolaev and B.G. Zakharov, Phys. Lett. B343, 13 (1995). 
[19] A. Bianconi, S. Jeschonnek, N.N. Nikolaev and B.G. Zakharov, Phys. Lett. B338, 123 (1994).

[20] F. Cannata, J.P. Dedonder and L. Lesniak, Phys. Rev. C33, 1888 (1986). 


\section{Captions}

Fig. 1 - The distorted spectral density $S_{\alpha}^{\mathrm{D}}$ as a function of the proton momentum $p$ for the ${ }^{12} \mathrm{C}\left(\mathrm{e}, \mathrm{e}^{\prime} \mathrm{p}\right){ }^{11} \mathrm{~B}_{\mathrm{s} 1 / 2}^{*}$ reaction in parallel kinematics at the momentum transfer $q=7 \mathrm{fm}^{-1}$. The dashed line is the result of the PWIA. The solid and the dotted lines are obtained in the framework of method I and II, respectively (see text). The curves labelled by (a) are produced with $U=100, W=0 \mathrm{MeV} / \mathrm{c}$, where $U, W$ are the real and the imaginary parts of the optical potential, respectively. The curves labelled by (b) are produced with $U=0, W=100 \mathrm{MeV} / \mathrm{c}$.

Fig. 2 - The same as in fig. 1, but the curves labelled by (a) are produced with $U=$ $20, W=50 \mathrm{MeV} / \mathrm{c}$, while the curves labelled by (b) are produced with $U=50, W=150$ $\mathrm{MeV} / \mathrm{c}$.

Fig. 3 - The $S_{\alpha}^{\mathrm{D}}$ as a function of the angle $\gamma$ between $\boldsymbol{q}$ and $\boldsymbol{p}$ for the ${ }^{12} \mathrm{C}\left(\mathrm{e}, \mathrm{e}^{\prime} \mathrm{p}\right){ }^{11} \mathrm{~B}_{\mathrm{s} 1 / 2}^{*}$ reaction in perpendicular kinematics at $q=7 \mathrm{fm}^{-1}$. The dashed line is the PWIA result, the dotted line is the pure contribution of the FSI (when the PWIA contribution is subtracted), the solid line is the coherent sum of the two. All the curves are obtained in the framework of method I with $U=50, W=150 \mathrm{MeV} / \mathrm{c}$.

Fig. 4 - The same as in fig. 3 but with the bound state described by a harmonic oscillator. The dashed line is the PWIA result, the solid and dotted lines are the total result for method I and II, respectively. 


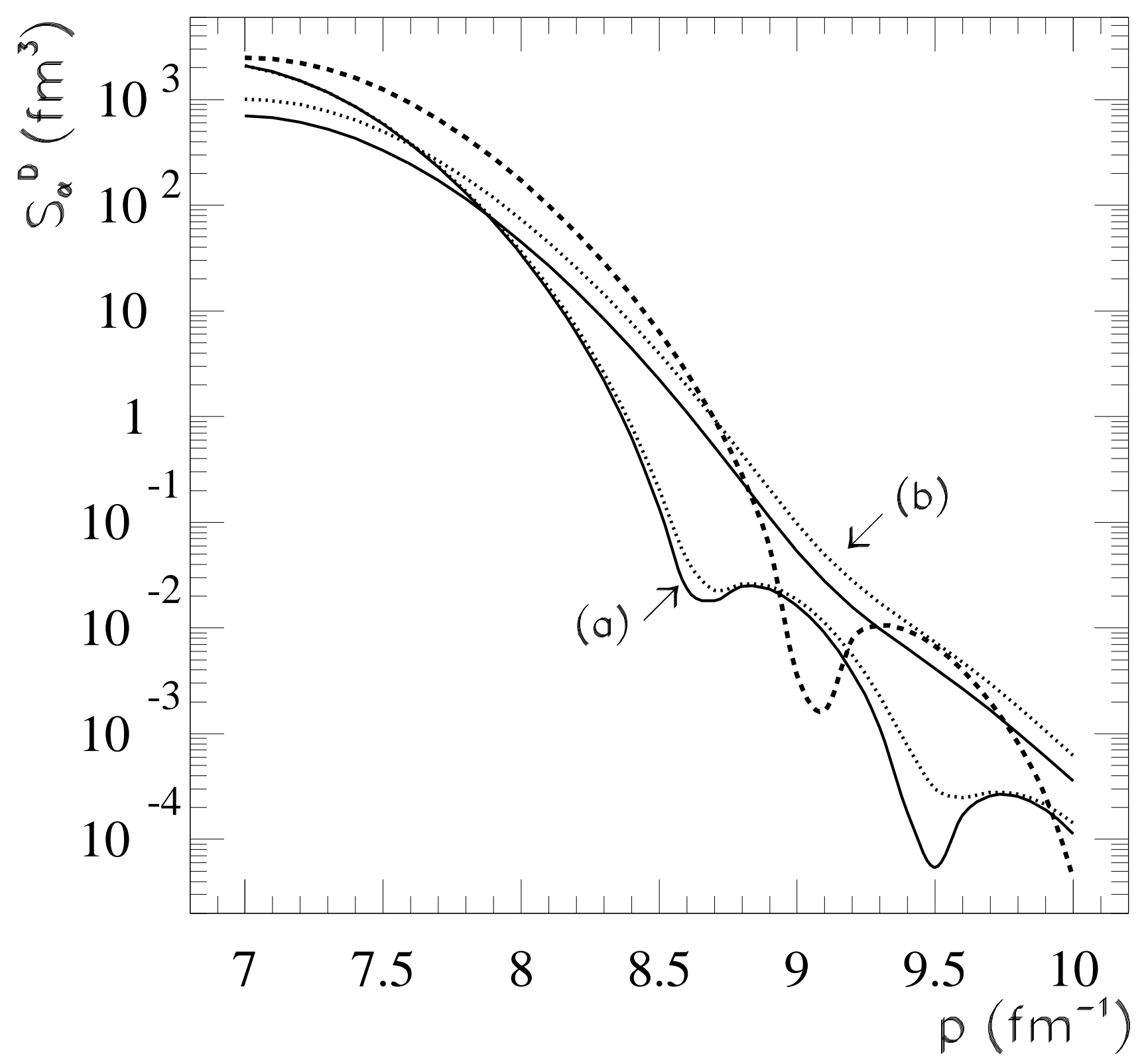




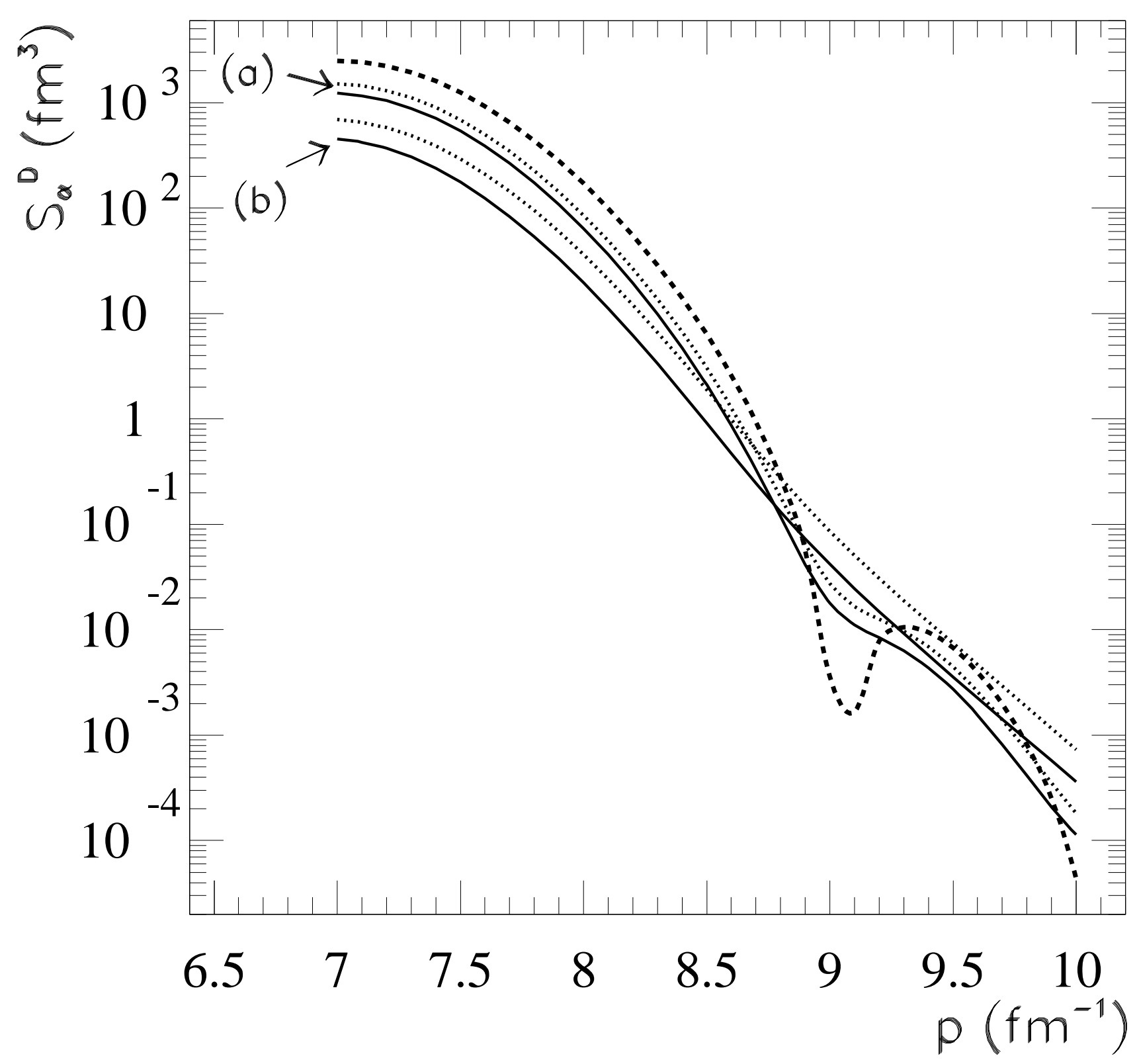




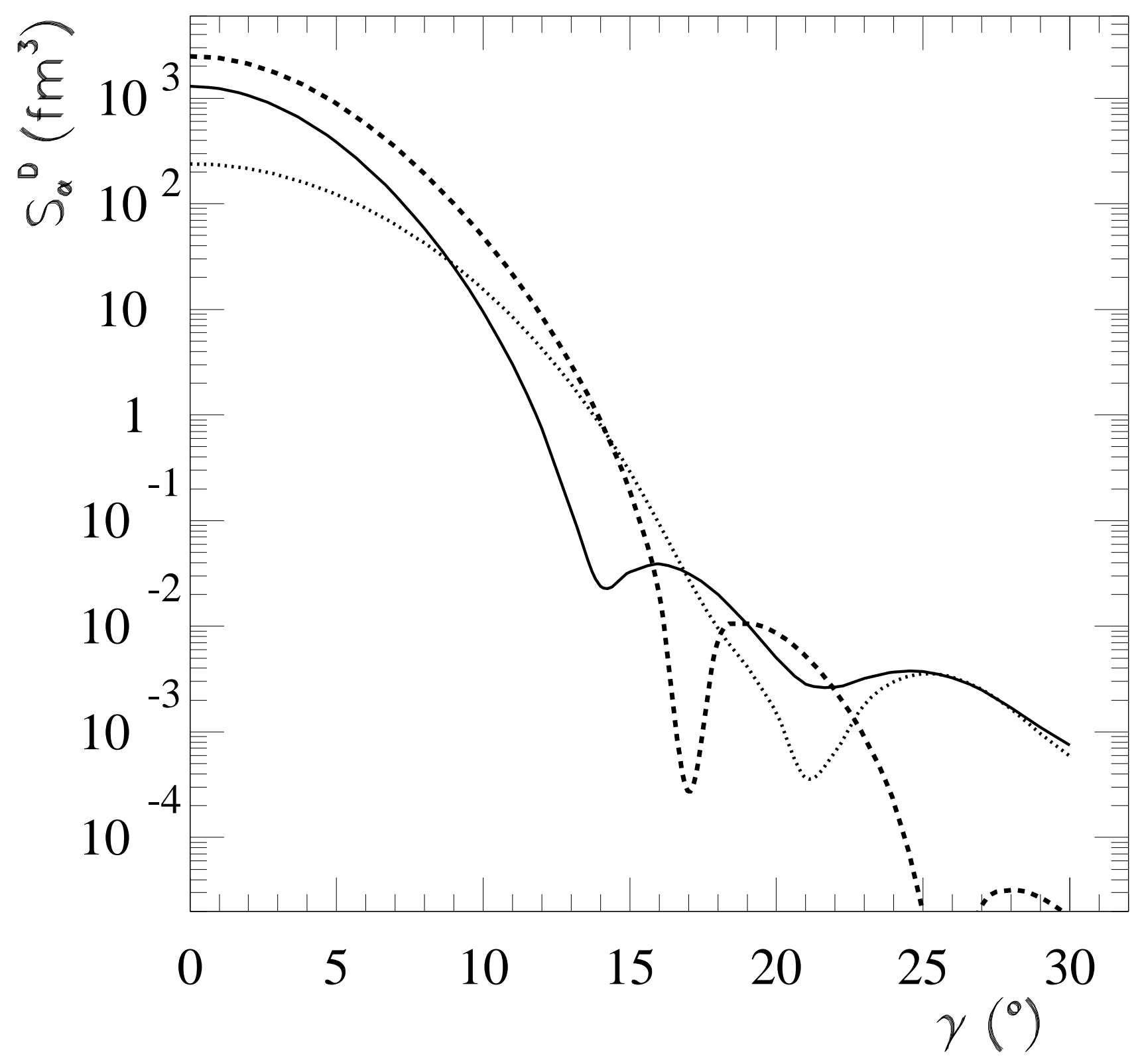




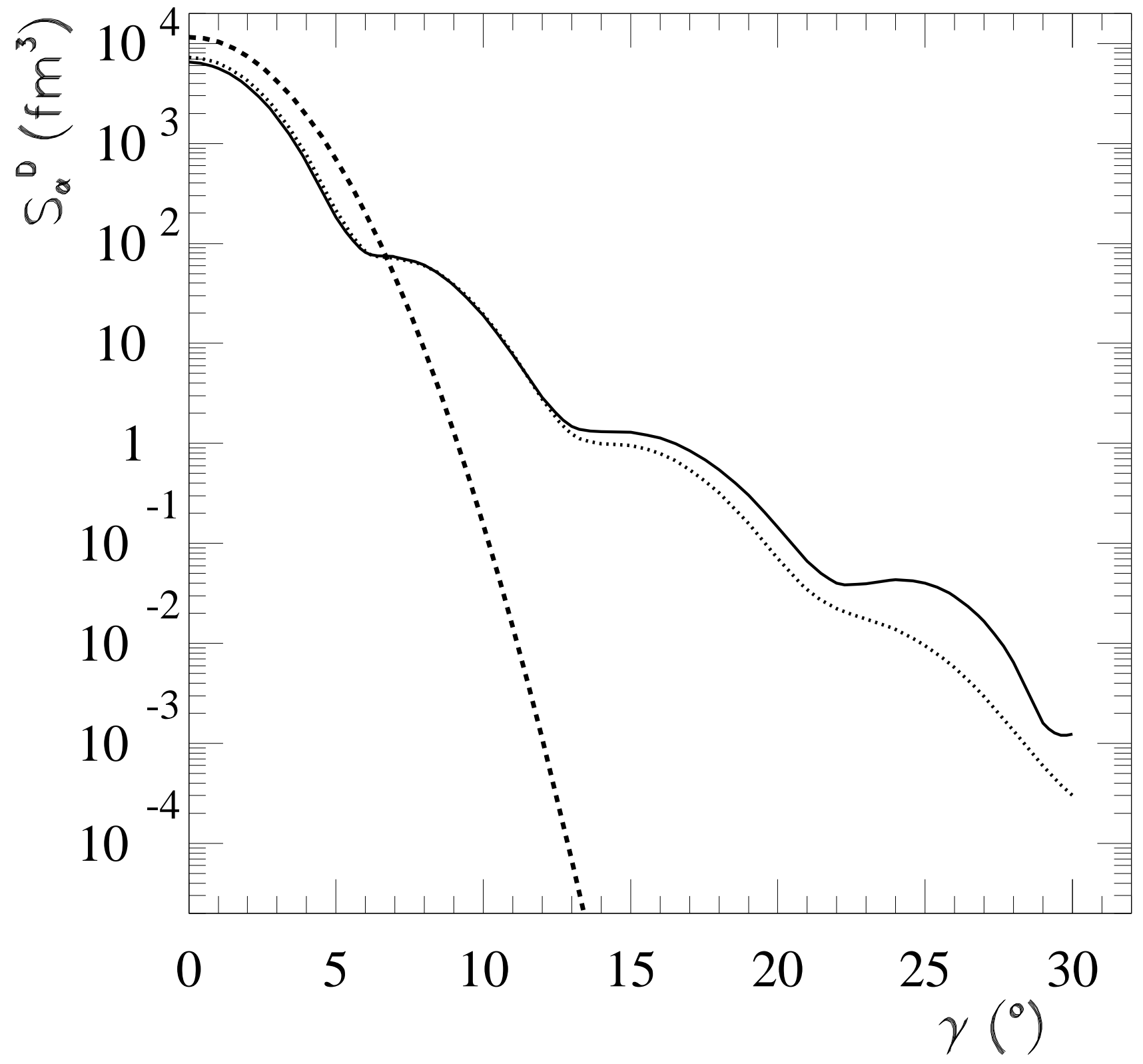

